\title{
THE PROBLEM OF ARTIFICIAL PRECISION IN THEORIES OF VAGUENESS: A NOTE ON THE RÔLE OF MAXIMAL CONSISTENCY
}

\author{
VINCENZO MARRA
}

\begin{abstract}
The problem of artificial precision is a major objection to any theory of vagueness based on real numbers as degrees of truth. Suppose you are willing to admit that, under sufficiently specified circumstances, a predication of "is red" receives a unique, exact number from the real unit interval $[0,1]$. You should then be committed to explain what is it that determines that value, settling for instance that my coat is red to degree 0.322 rather than 0.321. In this note I revisit the problem in the important case of Lukasiewicz infinite-valued propositional logic that brings to the foreground the rôle of maximally consistent theories. I argue that the problem of artificial precision, as commonly conceived of in the literature, actually conflates two distinct problems of a very different nature.
\end{abstract}

The monadic predicate $P(x):=$ " $x$ is prime", interpreted over the set of natural numbers $x \geqslant 1$, is (absolutely) precise: its extension is the set of prime numbers; its anti-extension is the set of composite numbers; each number either belongs to the extension of $P$ or to its anti-extension, but not to both; and in principle there is no issue as to whether a given number be prime or composite - though in practice it may be impossible to ascertain which is the case for an astronomic instance of $x$. By contrast, the monadic predicate $R(x):=$ " $x$ is red", interpreted over the set of all objects, is (to some extent) vague: its extension ought to be the set of all red objects; its anti-extension ought to be the set of all non-red objects; but it may not be clear, even in principle, just which objects do qualify as red, and which as non-red - think of a peculiar tint at the borderline between red and pink.

Is there a logic of vague predicates - or, for that matter, of vague propositions? Any definite answer, at present, is likely to be contentious. The philosophical literature on vagueness is relatively large; accounts of the main competing theories may be found in [Wil96, Kee00, Sha06, Smi08]. One cluster of theories is based on the assumption that any instantiation of a vague predicate $R$ by a constant $c$ whose denotatum lies, intuitively, at the borderline between the extension of $R$ and its complement, is only true to a degree. Thus if $c$ denotes my coat, and my coat is of a peculiar tint at the borderline between red and pink, on theories of this sort the proposition $R(c):=$ "My coat is red" is to be considered neither true nor false, but rather true to some intermediate degree. In this line of thought, a much stronger and yet popular assumption is that the real unit interval $[0,1]$ embodies "degrees of truth". See [Wil96, Chapter 4] for an account of many-valued approaches to vagueness; [Smi08] is a recent proposal of a theory of vagueness based on degrees of truth modelled by $[0,1]$. 
Arguably, any such $[0,1]$-valued theory of vagueness faces the problem of artificial precision. ${ }^{1}$ Perhaps the first passage were the locution 'artificial precision' was used in this connection is in [Haa79, p. 443]:

[Fuzzy logic] imposes artificial precision [... While] one is not obliged to require that a predicate either definitely applies or definitely does not apply, one is obliged to require that a predicate definitely applies to such-and-such, rather than to such-and-such other, degree (e.g. that a man $5 \mathrm{ft} 10 \mathrm{in}$ tall belongs to tall to degree 0.6 rather than 0.5).

Tye [Tye89, p. 14] makes a similar point:

One serious objection to [the many-valued approach] is that it really replaces vagueness with the most incredible and refined precision.

In these terms, the objection is rather generic. It is not clear that a precise semantics for vague concepts is, per se, a contradictory prospect. A sharper form of the objection, however, was put forth by Keefe [Kee00, p. 47], who identifies the source of the problem in our failure to see what could determine the correct value uniquely:

$[\mathrm{T}]$ he degree theorist's assignments impose precision in a form that is just as unacceptable as a classical true/false assignment. [...] All predications of "is red" will receive a unique, exact value, but it seems inappropriate to associate our vague predicate "red" with any particular exact function from objects to degrees of truth. For a start, what could determine which is the correct function, settling that my coat is red to degree 0.322 rather than 0.321 ?

Smith [Smi08, p. 279] endorses the objection in this form:

Intuitively, it is not correct to say that there is one unique element of $[0,1]$ that correctly represents the degree of truth of 'Bob is bald', with all other choices being incorrect. [...] we have an affront to intuition [because] [w]e cannot see what could possibly determine that the degree of truth of 'Bob is bald' is 0.61 rather than 0.62 or $0.6[\ldots]$

There is no question, I think, that the problem of artificial precision construed in this manner is a genuine objection to $[0,1]$-valued theories of vagueness; no such theory can get away without a plausible response to it. The purpose of this note is not to provide such a response. Rather more modestly, my aim is merely to point out that the problem of artificial precision actually conflates two distinct issues of a very different nature: one falls within the realm of mathematical logic proper; the other belongs to enquiries into the semantics of vagueness. It seems to me that being clear about this distinction is an important preliminary to any treatment of the problem of artificial precision.

The problem of artificial precision is only concerned with propositions, because only propositions can receive a truth value. In order to focus on the essence of the objection, I will therefore restrict attention to propositional logic throughout

\footnotetext{
${ }^{1}$ The related but distinct problem of higher-order vagueness (see e.g. [Kee00, pp. 31-36]) will not be considered in this note.
} 
the paper. However, even once it be agreed that truth values range in $[0,1]$, there remains much leeway to develop a formal system of many-valued propositional logic. The technical details of some of my arguments will depend on the specifics of the formal system under consideration; it is therefore important to be clear about the $[0,1]$-valued logic in question. I propose to concentrate on Eukasiewicz (infinitevalued propositional) logic. This is a non-classical system going back to the 1920's, cf. the early survey [ŁT30, §3], and its annotated English translation in [Tar56, pp. 38-59]. The standard modern reference for Łukasiewicz logic is [CDM00], while [Mun11] deals with topics at the frontier of current research. Łukasiewicz logic can also be regarded as a member of a larger hierarchy of many-valued logics that was systematised by Petr Hájek in the late Nineties, cf. [Háj98]. Here I recall the basic notions.

Let us fix once and for all the countably infinite set of propositional variables:

$$
\mathrm{VAR}=\left\{X_{1}, X_{2}, \ldots, X_{n}, \ldots\right\} .
$$

Let us write $\perp$ for the logical constant falsum, $\neg$ for the unary negation connective, and $\rightarrow$ for the binary implication connective. (Further derived connectives are introduced below.) The set Form of (well-formed) formulæ ${ }^{2}$ is defined exactly as in classical logic over the language $\{\perp, \neg, \rightarrow\}$.

The Eukasiewicz calculus is defined by the five ${ }^{3}$ axiom schemata

(A0) $\perp \rightarrow \alpha$

(A1) $\alpha \rightarrow(\beta \rightarrow \alpha)$

(A2) $(\alpha \rightarrow \beta) \rightarrow((\beta \rightarrow \gamma) \rightarrow(\alpha \rightarrow \gamma))$

(A3) $((\alpha \rightarrow \beta) \rightarrow \beta) \rightarrow((\beta \rightarrow \alpha) \rightarrow \alpha)$

(A4) $(\neg \alpha \rightarrow \neg \beta) \rightarrow(\beta \rightarrow \alpha)$
(Ex falso quodlibet.)

(A fortiori.)

(Implication is transitive.)

(Disjunction is commutative.)

(Contraposition.)

with modus ponens as the only deduction rule. Provability is defined exactly as in classical logic; $\vdash \alpha$ means that formula $\alpha$ is provable. I write $\mathscr{L}$ to denote Łukasiewicz logic.

The logical constant verum $(T)$, conjunction $(\wedge)$, disjunction $(\vee)$, and the biconditional $(\leftrightarrow)$ are defined as in Table 1. From the definition of disjunction one sees that (A3) indeed asserts the commutativity of disjunction. Other common derived connectives are reported in the same table, with their definition. Some remarks are in order. Using the biconditional, one defines formulæ $\alpha, \beta \in$ FORM to be logically equivalent just in case $\vdash \alpha \leftrightarrow \beta$ holds. The connectives $\odot$ and $\oplus$ are then De Morgan dual: $\alpha \oplus \beta$ is logically equivalent to $\neg(\neg \alpha \odot \neg \beta)$, and $\alpha \odot \beta$ is logically equivalent to $\neg(\neg \alpha \oplus \neg \beta)$. These connectives, known as the strong disjunction $(\oplus)$ and strong conjunction $(\odot)$ of $\mathscr{L}$, play a central rôle both in Hájek's treatment of many-valued logics [Háj98], and in Chang's algebraisation of $\mathscr{L}$ via MV-algebras [CDM00]. They are not idempotent, in the sense that $\alpha \oplus \alpha$ and $\alpha$ are not logically equivalent: only the implication $\alpha \rightarrow \alpha \oplus \alpha$ is provable; dual considerations apply to $\odot$. Conjunction $(\wedge)$ and disjunction $(\vee)$ also are De Morgan dual, but they

\footnotetext{
${ }^{2} \mathrm{~A}$ set of conventions for omitting parentheses in formulæ is usually adopted $(\perp$ is more binding than $\neg$, and $\neg$ is more binding than $\rightarrow$ ), and later extended to derived connectives. I do not spell the details here, as the conventions are analogous to the ones in classical logic, and are unlikely to cause confusion.

${ }^{3}$ In [CDM00, Chapter 4] the language has no logical constants, and consequently (A0) does not appear as an axiom. I prefer to explicitly have $\perp$ in the language, and thus I add Ex falso quodlibet to the standard axiomatisation.
} 


\begin{tabular}{|c|c|c|c|}
\hline Notation & Definition & Name & Idempotent \\
\hline \hline$\perp$ & - & Falsum & - \\
\hline$\top$ & $\neg \perp$ & Verum & - \\
\hline$\neg \alpha$ & - & Negation & - \\
\hline$\alpha \rightarrow \beta$ & - & Implication & - \\
\hline$\alpha \vee \beta$ & $(\alpha \rightarrow \beta) \rightarrow \beta$ & (Lattice) Disjunction & Yes \\
\hline$\alpha \wedge \beta$ & $\neg(\neg \alpha \vee \neg \beta)$ & (Lattice) Conjunction & Yes \\
\hline$\alpha \leftrightarrow \beta$ & $(\alpha \rightarrow \beta) \wedge(\beta \rightarrow \alpha)$ & Biconditional & - \\
\hline$\alpha \oplus \beta$ & $\neg \alpha \rightarrow \beta$ & Strong disjunction & No \\
\hline$\alpha \odot \beta$ & $\neg(\alpha \rightarrow \neg \beta)$ & Strong conjunction & No \\
\hline$\alpha \ominus \beta$ & $\neg(\alpha \rightarrow \beta)$ & But not, or Difference & - \\
\hline
\end{tabular}

TABLE 1. Connectives in Łukasiewicz logic.

are idempotent; in fact, they are sometimes called the lattice connectives because they induce the structure of a distributive lattice in the algebraic semantics of $\mathscr{L}$. Finally, the connective $\ominus$ is the co-implication, i.e. the dual to $\rightarrow$.

If $S \subseteq$ Form is any set of formulæ, one writes $S \vdash \alpha$ to mean that $\alpha$ is provable in Eukasiewicz logic, under the additional set of assumptions $S$. When this is the case, one says that $\alpha$ is a syntactic consequence of $S$. Since each one of (A0-A4) is a principle of classical reasoning, and since modus ponens is a classically valid rule of inference, each formula provable in $\mathscr{L}$ is a theorem of classical propositional logic. The converse is not true: most notably, the tertium non datur law, $\alpha \vee \neg \alpha$, is not provable in Łukasiewicz logic; this is one simple consequence of the completeness theorem to be recalled shortly. In fact, it can be shown that the addition of $\alpha \vee \neg \alpha$ as a sixth axiom schema to (A0-A4) yields classical logic.

By a theory in Eukasiewicz logic one means any set of formulæ that is closed under provability, i.e. is deductively closed. For any $S \subseteq$ Form, the smallest theory that extends $S$ exists: it is the deductive closure $S^{\vdash}$ of $S$, defined by $\alpha \in S^{\vdash}$ if, and only if, $S \vdash \alpha$. A theory $\Theta$ is consistent if $\Theta \neq$ Form, and inconsistent otherwise; and it is maximal, or maximally consistent, if it is consistent, and whenever $\alpha \in$ Form is such that $\alpha \notin \Theta$, then $(\Theta \cup\{\alpha\})^{\vdash}=$ Form, i.e. $\Theta \cup\{\alpha\}$ is inconsistent. A theory $\Theta$ is axiomatised by a set $S \subseteq$ Form of formula if it so happens that $\Theta=S^{\vdash}$; and $\Theta$ is finitely axiomatisable if $S$ can be chosen finite.

Let us now turn to the $[0,1]$-valued semantics. An atomic assignment, or atomic evaluation, is an arbitrary function $\bar{w}$ : VAR $\rightarrow[0,1]$. Such an atomic evaluation is uniquely extended to an evaluation of all formulæ, or possible world, i.e. to a function $w:$ FORM $\rightarrow[0,1]$, via the compositional rules:

$$
\begin{aligned}
w(\perp) & =0, \\
w(\alpha \rightarrow \beta) & =\min \{1,1-(w(\alpha)-w(\beta))\}, \\
w(\neg \alpha) & =1-w(\alpha) .
\end{aligned}
$$

It follows by trivial computations that the formal semantics of derived connectives is the one reported in Table 2. Tautologies are defined as those formulæ that evaluate to 1 under every evaluation. Let us write $\vDash \alpha$ to mean that the formula $\alpha \in$ Form is a tautology. The relativisation of this concept to theories leads to the notion of semantic consequence. Let $S \subseteq$ Form be any subset, and let $\Theta=S^{\vdash}$ be its 


\begin{tabular}{|c|c|}
\hline Notation & Formal semantics \\
\hline \hline$\perp$ & $w(\perp)=0$ \\
\hline$\top$ & $w(\top)=1$ \\
\hline$\neg \alpha$ & $w \alpha)=1-w(\alpha)$ \\
\hline$\alpha \rightarrow \beta$ & $w(\alpha \rightarrow \beta)=\min \{1,1-(w(\alpha)-w(\beta))\}$ \\
\hline$\alpha \vee \beta$ & $w(\alpha \wedge \beta)=\min \{w(\alpha), w(\beta)\}$ \\
\hline$\alpha \wedge \beta$ & $w(\alpha \leftrightarrow \beta)=1-|w(\alpha)-w(\beta)|$ \\
\hline$\alpha \leftrightarrow \beta$ & $w(\alpha \oplus \beta)=\min \{1, w(\alpha)+w(\beta)\}$ \\
\hline$\alpha \oplus \beta$ & $w(\alpha \odot \beta)=\max \{0, w(\alpha)+w(\beta)-1\}$ \\
\hline$\alpha \odot \beta$ & $w(\alpha \ominus \beta)=\max \{0, w(\alpha)-w(\beta)\}$ \\
\hline$\alpha \ominus \beta$ &
\end{tabular}

TABLE 2. Formal semantics of connectives in Łukasiewicz logic.

associated theory. Given $\alpha \in$ Form, the assertion $S \vDash \alpha$ states that any evaluation $w:$ Form $\rightarrow[0,1]$ that satisfies $w(S)=\{1\}$ - meaning that $w(\beta)=1$ for each $\beta \in S-$ must also satisfy $w(\alpha)=1$. When this is the case, we say that $\alpha$ is a semantic consequence of $S$. We write $S^{\models}$ for the set of semantic consequences of $S$.

It is an exercise to check that $\mathscr{L}$ enjoys the generalised validity theorem: for any $S \subseteq$ Form and any $\alpha \in$ Form, if $S \vdash \alpha$ then $S \models \alpha$. On the other hand, it is a non-trivial theorem that $\mathscr{L}$ is complete ${ }^{4}$ with respect to the many-valued semantics above: hence $\vdash \alpha$ if, and only if, $\models \alpha$, for any $\alpha \in$ Form. The first proof of this appeared in [RR58]; see also [CDM00, 4.5.1 \& 4.5.2].

All of the above can be adapted in the obvious manner to the finite set $\mathrm{VAR}_{n}=$ $\left\{X_{1}, \ldots, X_{n}\right\}$, in which case one speaks of Łukasiewicz logic over $n$ (propositional) variables, denoted $\mathscr{L}_{n}$. Although, strictly speaking, one should introduce fresh consequence relation symbols $\vdash_{n}$ and $\vDash_{n}$ for each $\mathscr{L}_{n}$, I will avoid this pedantry and use $\vdash$ and $\vDash$ in all cases; context will do the rest. I will write $\mathrm{FORM}_{n}$ for the set of formulæ whose propositional variables are contained in $\mathrm{VAR}_{n}$. We will be mostly concerned with the apparently trivial case of $\mathscr{L}_{1}$.

\section{3}

What is the logical status of an assumption such as "VM is tall" is true to degree $r \in[0,1]$ "? Let us begin with the remark of an eminent logician [Hó9, p. $368]$.

[Regarding the problem of artificial precision,] [1]et us comment that mathematical fuzzy logic concerns the possibility of sound inference, surely not techniques of ascribing concrete truth degrees to concrete propositions.

\footnotetext{
${ }^{4}$ However, $\mathscr{L}$ fails strong completeness (i.e. completeness for theories): there is a set $S \subseteq$ Form and a formula $\alpha \in$ Form such that $S \models \alpha$, but $S \forall \alpha$; see [CDM00, 4.6].

${ }^{5}$ Not all vague predicates are alike. The predicate Tall $(\cdot)$ comes in pair with its opposite, Short $(\cdot)$, over the domain of all individuals, say; but Red $(\cdot)$ does not: there is no colour term for non-Red $(\cdot)$ in the visible spectrum. Although I will not argue the point in this note, I believe that $\mathscr{L}$ cannot be an appropriate formal model of vague predicates such as Red (.). Hence the shift from redness to tallness.
} 
The point Hájek is making here is by no means limited to mathematical fuzzy logic. Logic is exclusively concerned with the form of an argument, not with its content. Logic can teach us nothing (factual).

Thus the assumption "VM is tall' is true to degree $r$ " has extra-logical content. In particular, it is a semantic assumption: it tells us that certain states of affairs (namely, those wherein "VM is tall" is true to a degree $\neq r$ ), while perhaps logically consistent, are known (or assumed) not to be the case. In fact, it is reasonable to expect that the assumption "VM is tall" is true to degree $r$ " be maximally strong, falling short only of the strongest, inconsistent assumption according to which everything is the case. For the stronger an assumption is, the fewer models it has, i.e. the fewer are the possible worlds that are consistent with it. Now the assumption " VM is tall' is true to degree $r$ " leaves us with just one $e^{6}$ possible world consistent with it, namely, the one world in which VM is tall to degree exactly $r$. This solitary possible world is the bare minimum we need to stay clear of the precipice of inconsistency.

We can considerably sharpen these initial remarks. Let us focus on the vague proposition

$$
X_{1}:=\text { "VM is tall", }
$$

formally modelled by the propositional variable $X_{1}$ in $\mathscr{L}_{1}$. There are two distinct situations.

(S1) All we know about $X_{1}$ is that it is a propositional variable.

(S2) All we know about $X_{1}$ is that it is a propositional variable such that the possible worlds $w:$ Form $\rightarrow[0,1]$ that we are ready to admit in light of the intended interpretation $\left(^{*}\right)$ of $X_{1}$ are precisely those satisfying $w\left(X_{1}\right)=r$, for a fixed real number $r \in[0,1]$.

Failure to distinguish between (S1) and (S2) amounts to ignoring a set of extralogical assumptions, namely, those encoded by $(*)$. Suppose first we are in situation (S1). Consider any formula $\alpha\left(X_{1}\right) \in \mathrm{FORM}_{1}$. Then, in our intended interpretation, $\alpha\left(X_{1}\right)$ is a statement about VM's tallness. But, given (S1), such a statement can be truthfully asserted if, and only if, it is a provable formula: $\vdash \alpha\left(X_{1}\right)$. In other words, such statements coincide with analytic truths (relative to $\mathscr{L}_{1}$ ) which, by their very nature, are absolutely uninformative about whoever's tallness. Given (S1) only, whatever statement one can truthfully assert about VM's tallness, one can equally truthfully assert about TW's thinness, and conversely. In situation (S1) the problem of artificial precision does not even make sense: there is no specific truth value to be puzzled about. ${ }^{7}$

By contrast, it is in situation (S2) that the problem of artificial precision arises. Now we are only concerned with evaluations $w:$ Form $\rightarrow[0,1]$ that satisfy $w\left(X_{1}\right)=$ $r$. Let us call such evaluations admissible ${ }^{8}$ for our intended interpretation $\left(^{*}\right)$ of $X_{1}$. Consider any formula $\alpha\left(X_{1}\right) \in \mathrm{FORM}_{1}$. Then, on our intended interpretation,

\footnotetext{
${ }^{6}$ Assuming that all propositions under consideration are built from the single atomic one "VM is tall", and that the logic is truth-functional. Since, formally, the discussion applies to $\mathscr{L}_{1}$, these assumptions are satisfied.

${ }^{7}$ Compare Haack's claim that "[In fuzzy logic] one is obliged to require that a predicate definitely applies to such-and-such, rather than to such-and-such other, degree" [Haa79, loc. cit.]. It was just shown that the claim, if taken at face value, is unwarranted.

${ }^{8}$ There is just one such admissible evaluation in $\mathscr{L}_{1}$, of course. I am using the plural form in preparation for the forthcoming extension $\left(\mathrm{S}_{T}\right)$.
} 
$\alpha\left(X_{1}\right)$ is a statement about VM's tallness. But, given (S2), it is no longer the case that $\alpha\left(X_{1}\right)$ can be truthfully asserted if, and only if, $\vdash \alpha\left(X_{1}\right)$. On the one hand, if $\vdash \alpha\left(X_{1}\right)$, then certainly any admissible $w$ satisfies $w\left(\alpha\left(X_{1}\right)\right)=1$ by the validity theorem, so that $\alpha\left(X_{1}\right)$ is indeed true on our intended interpretation $\left(^{*}\right)$ - but again, such analytic truths have nothing to do with my tallness. On the other hand, however, there will be formulæ $\alpha\left(X_{1}\right)$ that are not provable in $\mathscr{L}_{1}$, but are such that $\alpha\left(X_{1}\right)$ can be truthfully asserted under (S2), precisely because we restrict attention to admissible evaluations only. For a trivial example, assume $r=1$ : then the formula $X_{1}$ can be truthfully asserted subject to (S2), simply because we restrict attention to the one possible world where $X_{1}$ indeed holds. In general, let $\Theta_{r} \subseteq$ FORM $_{1}$ be the collection of all those formulæ over the variable $X_{1}$ that may be truthfully asserted given (S2), that is, set

$$
\Theta_{r}=\left\{\alpha\left(X_{1}\right) \in \text { FoRm }_{1} \mid w\left(\alpha\left(X_{1}\right)\right)=1 \text { whenever } w\left(X_{1}\right)=r\right\},
$$

where $w:$ Form $_{1} \rightarrow[0,1]$ ranges over all possible worlds. Then those formulæ in $\Theta_{r}$ that are not analytic truths are precisely the synthetic, factual truths about VM's tallness that the semantic assumption $w\left(X_{1}\right)=r$ entails, and that $\mathscr{L}_{1}$ is able to express syntactically. In other words, the set of formulæ $\Theta_{r}$ attempts to encode the semantic assumption (S2) at the syntactic level, to within the formal linguistic resources afforded by $\mathscr{L}_{1}$.

There are intermediate situations, of course.

$\left(\mathrm{S}_{T}\right)$ All we know about $X_{1}$ is that it is a propositional variable such that the possible worlds $w:$ FORM $\rightarrow[0,1]$ that we are ready to admit in light of the intended interpretation $\left(^{*}\right)$ of $X_{1}$ are precisely those satisfying $w\left(X_{1}\right) \in T$, for a fixed subset $T \subseteq[0,1]$.

If $T=[0,1]$, then $\left(\mathrm{S}_{T}\right)$ is $(\mathrm{S} 1)$ : we are imposing no restriction on possible worlds. If $T=\{r\}$, then $\left(\mathrm{S}_{T}\right)$ is $(\mathrm{S} 2)$ : the only possible world we are ready to admit is the one with $w\left(X_{1}\right)=r$. We can adapt $(\dagger)$ to the intermediate situations in the obvious manner:

$$
\Theta_{T}=\left\{\alpha\left(X_{1}\right) \in \text { Form }_{1} \mid w\left(\alpha\left(X_{1}\right)\right)=1 \text { whenever } w\left(X_{1}\right) \in T\right\},
$$

where $w:$ FoRM $_{1} \rightarrow[0,1]$ ranges over all possible worlds.

It turns out that $\Theta_{T}$ as in $(\ddagger)$ is a theory in $\mathscr{L}_{1}$ for any choice of $T \subseteq[0,1]$ : the generalised validity theorem guarantees that the semantic assumption $\left(\mathrm{S}_{T}\right)$ is reflected into a deductively closed set of syntactic assumptions ( $\ddagger)$. It is also clear by the very definition $(\ddagger)$ that $\Theta_{T}$ is a superset of $\Theta_{T^{\prime}}$ whenever $T \subseteq T^{\prime}$, for any two subsets $T, T^{\prime} \subseteq[0,1]$. Thus the (syntactic representation of the) assumption, say, " $\mathrm{VM}$ is tall' is true to degree $\geqslant \frac{1}{2}$ " is no stronger than the (syntactic representation of the) assumption " VM is tall' is true to degree $\frac{2}{3}$ ". Once more, this suggests that a theory $\Theta_{r}$, for $r \in[0,1]$, should correspond to a maximally strong assumption.

Let now $\mathscr{M}$ be the set of all theories in $\mathscr{L}_{1}$ that can be written in the form (†). That is,

$$
\mathscr{M}=\left\{\Sigma \subseteq \text { FoRm }_{1} \mid \text { There exists } r \in[0,1] \text { such that } \Sigma=\Theta_{r}\right\} .
$$

The next proposition ${ }^{9}$ confirms the intuitions above about the members of $\mathscr{M}$.

\footnotetext{
${ }^{9}$ As mentioned, $\Theta_{r}$ is deductively closed for any $r \in[0,1]$. Given $\alpha \in$ Form $_{1}$, suppose $\Theta_{r} \vdash \alpha$. If $w_{r}:$ FoRm $_{1} \rightarrow[0,1]$ is the unique evaluation such that $w_{r}\left(X_{1}\right)=r$, then $w_{r}\left(\Theta_{r}\right)=\{1\}$ by $(\dagger)$; since $\Theta_{r} \vdash \alpha$, then $w_{r}(\alpha)=1$ by the generalised validity theorem for $\mathscr{L}_{1}$; hence $\alpha \in \Theta_{r}$, again by
} 
Proposition 1. $\mathscr{M}$ is precisely the collection of all maximally consistent theories in $\mathscr{L}_{1}$.

This result leaves open the possibility that different real numbers determine the same maximally consistent theory via $(\dagger)$, which leads to a key question.

(Q1) Is the semantic assumption (S2) precisely equivalent to the set of syntactic assumptions $(\dagger)$ ? More precisely, is the correspondence

$$
r \in[0,1] \longmapsto \Theta_{r} \in \mathscr{M}
$$

determined by definition $(\dagger)$ a bijection between the real unit interval $[0,1]$, and the set $\mathscr{M}$ of maximally consistent theories in $\mathscr{L}_{1}$ ?

I note in passing that generalisations of this question make sense for arbitrary sets of propositional variables ${ }^{10}$ (i.e. for $\mathscr{L}_{n}, n>0$ an integer, and for the whole of $\mathscr{L}$ ), and, in another direction, for arbitrary subsets $T \subseteq[0,1]$ of truth values and their associated theories ${ }^{11}$ as in $(\ddagger)$. However, for the purposes of this note it will be enough to concentrate on (Q1).

Although the details will vary, ${ }^{12}$ close analogues of (Q1) can be asked for virtually any $[0,1]$-valued logic $\mathscr{S}$, under rather weak assumptions. If the answer to (Q1) is negative then there is a discrepancy between our formal, $[0,1]$-valued semantics, and the expressive power of such a logic $\mathscr{S}$. For if $\Theta_{r}=\Theta_{s}$ with $r \neq s \in[0,1]$, then the logic $\mathscr{S}$ is not sufficiently expressive to discern between $r$ and $s$, so that it is legitimate to ask for further support to the claim that real numbers are the basis of a suitable formal semantics for $\mathscr{S}$. Indeed, proving that (Q1) has negative answer for a specific [0,1]-valued logic is a way of making precise the assertion that its $[0,1]$-valued semantics is "redundant". I do not wish to suggest that all redundant formal semantics (in the present sense) is useless. What I am implying, though, is that there is a sense in which a redundant semantics poses a challenge to logicians: some justification for redundancy ought to be given, lest one incurs Occam's razor. ${ }^{13}$

(†). Moreover, $\Theta_{r}$ is consistent: since $w_{r}(\perp)=0$ by the semantics of $\perp$, we have $\perp \notin \Theta_{r}$ in light of $(\dagger)$. It is harder to prove that $\Theta_{r}$ is maximally consistent, and that all maximally consistent theories are of this form. However, this is a standard result (essentially [CDM00, 4.6.3 and 3.5.1]).

${ }^{10}$ In which case Proposition 1 and question (Q1) would be concerned with maximally consistent theories in $\mathscr{L}_{n}$ or $\mathscr{L}$.

${ }^{11}$ In which case Proposition 1 and question (Q1) would be concerned with semisimple theories, i.e. those theories for which completeness holds; see [CDM00, 4.6 and 3.6].

${ }^{12}$ The main issue in generalizing (Q1) to other systems is that Proposition 1 most often fails, so that it is not enough to consider maximally consistent theories only. For example, in the important Gödel-Dummett logic [Háj98, Chapter 4], $\mathscr{M}$ turns out to be exactly the collection of all prime theories in the one-variable fragment, where a theory $\Theta$ is prime if it proves either $\alpha \rightarrow \beta$ or $\beta \rightarrow \alpha$ for any two formulæ $\alpha$ and $\beta$.

${ }^{13}$ If $\mathscr{S}$ is such that (Q1) has negative answer, then the formal semantics of $\mathscr{S}$ violates Leibniz's Identity of Indiscernibles: in deference to which, we ought to identify real numbers (=possible worlds) $r, s \in[0,1]$ whenever they satisfy $\Theta_{r}=\Theta_{s}$, provided all we are concerned with are those properties of $r$ and $s$ that are expressible within $\mathscr{S}$. If we insist not to identify $r$ and $s$, then there must be distinguishing properties of these two possible worlds, not expressible with the linguistic resources of $\mathscr{S}$, that we nonetheless wish our formal semantics to record. A formal semantics strictly richer than the available language is of course a perfectly reasonable construct, but it had better result from an explicit choice - not from overlooking a negative answer to (Q1). I do not elaborate this line of thought further in this note. 
Such worries need not concern us insofar as we are dealing with Łukasiewicz logic:

Proposition 2. The answer to (Q1) is affirmative. That is, ( $\star$ ) is a bijection between $[0,1]$ and maximally consistent theories in $\mathscr{L}_{1}$.

This fact ${ }^{14}$ rests on Otto Hölder's theorem from 1901 that a totally ordered Archimedean group embeds into the real numbers. The mathematical details involved should not blind us to the remarkable conceptual content of Proposition 2: the innocent-looking axioms (A0-A4) of Eukasiewicz logic characterise the real numbers, in the sense that maximally consistent theories in $\mathscr{L}_{1}$ classify the elements of $[0,1]$.

What does all this have to do with vagueness? Nothing: (Q1) does not mention vagueness at all. Mutatis mutandis, (Q1) is a question that can be asked about virtually any logic with a [0,1]-valued semantics, and the answer will be independent of any intended interpretation of that logic; briefly, (Q1) is a question in mathematical logic.

But, having isolated (Q1) as worthy of attention, we can proceed to ask a second key question.

(Q2) Given $r \in[0,1]$, can we read off $\Theta_{r}$ as in ( $\dagger$ ) the synthetic truths about $X_{1}$ determined by (S2) in a form that is intelligible with respect to our intended interpretation $(*)$ ?

Vagueness - the intended interpretation - enters the picture at this stage: for (Q2) makes no sense in terms of the formal semantics alone. We are given the truth value $r \in[0,1]$, and the corresponding maximally consistent theory $\Theta_{r}$ induced in $\mathscr{L}_{1}$ by $(\dagger)$. We must provide, if we can, an interpretation of the formulæ in $\Theta_{r}$ as assertions of vague propositions about VM's tallness, that taken together amount to an explanation of what it means for VM to be tall to degree $r$. The answer to (Q2) is affirmative insofar as this interpretation and the ensuing explanation are convincing. In case $\Theta_{r}$ is finitely (hence singly) axiomatisable, and one such axiomatisation is known, ${ }^{15}$ we are thus concerned with a single formula $\alpha\left(X_{1}\right)$ such that $\Theta_{r}=\left\{\alpha\left(X_{1}\right)\right\}^{\vdash}$. We must provide a reading of $\alpha\left(X_{1}\right)$, as a single assertion about VM's tallness, that convincingly explains what it means to assume " $\mathrm{VM}$ is tall' is true to degree $r$ ". Observe that this explanation, whatever it is, need not (should not) mention $r$ itself - nor, for that matter, any other number. This is because $\alpha\left(X_{1}\right)$ is a formula in $\mathscr{L}_{1}$, and this logic has no truth constants other than verum and falsum.

\footnotetext{
${ }^{14}$ Algebraically, Proposition 2 amounts to the representation theorem for 1-generated semisimple MV-algebras, see [CDM00, Chapter 3]. Via Mundici's categorical equivalence between MValgebras and lattice-ordered Abelian groups with a strong order unit [CDM00, 7.1], this is equivalent to Hölder's theorem, for which the interested reader may consult [BKW77, §2.6].

${ }^{15}$ It can be proved that, given $r \in[0,1], \Theta_{r}$ as in $(\dagger)$ is finitely axiomatisable in $\mathscr{L}_{1}$ if, and only if, $r$ is rational. Moreover, it is possible to exhibit an algorithm (for definiteness, a Turing machine) that, on input any rational number $r \in[0,1]$, outputs a formula $\alpha_{r}\left(X_{1}\right)$ satisfying $\Theta_{r}=\left\{\alpha_{r}\left(X_{1}\right)\right\}^{\vdash}$. Everything hinges on the theory of continued fractions and Schauder hats; see [CDM00, Chapter 3].
} 
I suggest here that (Q2) is a sharper formulation of the problem of artificial precision, as stated at the beginning of this note, for Lukasiewicz logic - and in fact, the specifics aside, for any aspiring $[0,1]$-valued logic of vagueness.

So what is the answer to (Q2) for Eukasiewicz logic? New research ${ }^{16}$ is needed, I think, to say something defensible in this connection. Perhaps a glimpse of the difficulties involved may be caught if, by way of an epilogue to this note, we work our way up to the modestly non-classical truth value $\frac{1}{2}$.

The theory $\Theta_{1}$ is axiomatised by the single formula $X_{1}$. In symbols,

$$
\Theta_{1}=\left\{X_{1}\right\}^{\vdash} \text {. }
$$

In this case, the answer to (Q2) is that Your ${ }^{17}$ assumption " $\mathrm{VM}$ is tall' is true to degree 1" amounts to the fact that You are ready to assert that VM is clearly, indisputably tall.

Similarly, $\Theta_{0}$ is axiomatised by the single formula $\neg X_{1}$ :

$$
\Theta_{0}=\left\{\neg X_{1}\right\}^{\vdash} .
$$

The answer to (Q2) is clear in this case, too. The assumption " $\mathrm{VM}$ is tall' is true to degree 0" amounts to the fact that You are ready to assert that VM is clearly, indisputably non-tall: in short, short. ${ }^{18}$

What about $\Theta_{\frac{1}{2}}$ ? We have

$$
\Theta_{\frac{1}{2}}=\left\{\left(\neg X_{1} \rightarrow X_{1}\right) \wedge\left(X_{1} \rightarrow \neg X_{1}\right)\right\}^{\vdash} .
$$

Can we make sense of this? Quite generally (cf. Table 2), to assert $\alpha \wedge \beta$ in $\mathscr{L}$ is to assert $\alpha$ and to assert $\beta$; and to assert $\alpha \rightarrow \beta$ is to assert that $\alpha$ is (clearly, indisputably) less true ${ }^{19}$ than $\beta$, or at the very most just as true.

So Your assumption that " $\mathrm{VM}$ is tall' is true to degree $\frac{1}{2}$ " amounts to the fact that You are ready to assert both that VM is clearly, indisputably less of a case of a short man, than he is a case of a tall man, and that VM is clearly, indisputably less of a case of a tall man, than he is a case of a short man.

\footnotetext{
${ }^{16}$ Since [Mac76], Eukasiewicz logic has been widely discussed in the philosophical literature as a candidate for a logic of vagueness. More often than not, it has been rejected; cf. e.g. [Wil96, Kee00]. To the best of my knowledge, though, the quite specific question (Q2) has not been addressed.

${ }^{17}$ Compare Bruno de Finetti's usage [dF90a, dF90b] of the capitalised second person singular pronoun to stress that attributing a degree of probability to a (classical) proposition is a personal matter.

${ }^{18}$ Cf. Footnote 5 .

${ }^{19}$ Caution: no circularity is involved in this passage. The objection of artificial precision can only be raised against theories that (i) have already committed to degrees of truth, and (ii) have embraced $[0,1]$, or some other precisely specified structure, as a mathematical model for degrees of truth and their relationships. The charge that we are here using a comparative notion of truth to explain artificial precision, without justifying the assumption that truth does come in degrees, has therefore no force. Similary, the problem of justifying why degrees of truth are modelled by the real numbers rather than, say, the octonions, may well be a problem - there is no paucity of objections to (i-ii) in the literature - but it is a different one.
} 


\section{ACKNOWLEDGEMENTS}

A preliminary version of parts of this paper was presented at the meeting Epistemic Aspects of Many-Valued Logics, held in Prague at the Institute of Philosophy of the Academy of Sciences of the Czech Republic, from the $13^{\text {th }}$ to the $16^{\text {th }}$ of September 2010. I am grateful to the organisers, Timothy Childers, Christian Fermüller, and Ondrej Majer, for having given me a chance to present some of these ideas before an audience that included several philosophers who have thought deeply about vagueness. I am indebted to many participants for questions, discussions, and criticism that have been helpful in improving my initial ideas on the subject matter of this paper. In this connection, I should particularly like to thank Christian Fermüller, Colin Howson, Nicholas J. J. Smith, and Timothy Williamson.

\section{REFERENCES}

[BKW77] Alain Bigard, Klaus Keimel, and Samuel Wolfenstein, Groupes et anneaux réticulés, Lecture Notes in Mathematics, Vol. 608, Springer-Verlag, Berlin, 1977.

[CDM00] Roberto L. O. Cignoli, Itala M. L. D'Ottaviano, and Daniele Mundici, Algebraic foundations of many-valued reasoning, Trends in Logic - Studia Logica Library, vol. 7, Kluwer Academic Publishers, Dordrecht, 2000.

[dF90a] Bruno de Finetti, Theory of probability. Vol. 1, Wiley Classics Library, John Wiley \& Sons Ltd., Chichester, 1990. Reprint of the 1974 translation.

[dF90b] , Theory of probability. Vol. 2, Wiley Classics Library, John Wiley \& Sons Ltd., Chichester, 1990. Reprint of the 1975 translation.

[Hó9] Petr Hájek, On vagueness, truth values and fuzzy logics, Studia Logica 91 (2009), no. 3, 367-382.

[Haa79] Susan Haack, Do we need "fuzzy logic"?, Internat. J. Man-Mach. Stud. 11 (1979), no. 4, 437-445, Special issue on fuzzy reasoning (Workshop, Queen Mary Coll., London, 1978).

[Háj98] Petr Hájek, Metamathematics of fuzzy logic, Trends in Logic-Studia Logica Library, vol. 4, Kluwer Academic Publishers, Dordrecht, 1998.

[Kee00] Rosanna Keefe, Theories of vagueness, Cambridge University Press, Cambridge, 2000.

[ŁT30] Jan Łukasiewicz and Alfred Tarski, Untersuchungen über den Aussagenkalkül., C. R. Soc. Sc. Varsovie 23 (1930), 30-50.

[Mac76] Kenton F. Machina, Truth, belief, and vagueness, Journal of Philosophical Logic 5 (1976), no. 1, 47-78.

[Mun11] Daniele Mundici, Advanced Eukasiewicz Calculus and MV-algebras, Trends in LogicStudia Logica Library, vol. 35, Springer, New York, 2011.

[RR58] Alan Rose and J. Barkley Rosser, Fragments of many-valued statement calculi, Trans. Amer. Math. Soc. 87 (1958), 1-53.

[Sha06] Stewart Shapiro, Vagueness in context, Oxford University Press, 2006.

[Smi08] Nicholas J. J. Smith, Vagueness and degrees of truth, Oxford University Press, Oxford, 2008.

[Tar56] Alfred Tarski, Logic, semantics, metamathematics. Papers from 1923 to 1938, Oxford at the Clarendon Press, 1956, Translated by J. H. Woodger.

[Tye89] Michael Tye, Supervaluationism and the law of excluded middle, Analysis 49 (1989), no. $3,141-143$.

[Wil96] Timothy Williamson, Vagueness, Routledge, 1996.

Dipartimento di Matematica Federigo Enriques, Università degli Studi di Milano, via Cesare Saldini 50, 20133 Milano, Italy

E-mail address: vincenzo.marra@unimi.it 